\title{
In Situ Materials Characterization of 2-Dim Materials at High Energy and Spatial Resolution
}

\author{
Xuan Hu, ${ }^{1}$ Jacob R. Jokisaari, ${ }^{1}$ Liang Hong ${ }^{1}$ and Robert F. Klie ${ }^{1}$ \\ 1. University of Illinois at Chicago, Department of Physics, Chicago, IL.
}

Two-dimensional materials, including graphene, BN and transition metal dichalcogenides (TMDs), exhibit great potential for a variety of applications, such as transistors, spintronics, photovoltaics, or even as window layers for in-situ microscopy holders.[1,2] When 2-dim materials are used in electronic devices, the potential for miniaturization offers remarkable improvements in electrical performance. Yet, the effects of heat dissipation can be a problem in designing electronic devices. Therefore, the thermal properties of 2-dim materials are an important subject of current research and new methods are being developed for temperature measurements at the nanometer scale.[3] In TEM in-situ liquid holders that utilized graphene as a window layer, it was demonstrated that graphene interacts strongly with the contained liquid, which can be beneficial in reducing electron beam induced damage.[4] However, the strong interaction between the reaction species with the graphene window layer can potentially alter the chemistry within the liquid cell and might yield effects not observed in more conventional reactor setups.

Over the last few years, we a new approach that now enable us to measure the local temperature distribution and thermal expansion coefficient of 2-dim materials with nanometer resolution.[4] In addition, we have developed new, more inert window layers for liquid cells based on 2-dim materials that will enable in-situ characterization of fluids at unprecedented spatial and energy resolution. The electronbeam induced change in the $\mathrm{pH}$ of liquids, such as $\mathrm{H}_{2} \mathrm{O}$ will be demonstrated using the dissolution and precipitation of calcium hydroxyapatite in a liquid cell based on 2-dim material. The experiments are carried out using the aberration-corrected, cold-field emission JEOL ARM200CF at the University of Illinois at Chicago. The benefits monochromated STEM, such as the NION HERMES, on the characterization liquids encapsulated by 2-dim materials will also be discussed.

Our nanoscale thermometry measurements are based on the principle that the plasma peak position is sensitive to the density of charge carriers, which changes as a materials expands/contracts as a function of temperature. For 2-dim materials, we have shown that the thermal expansion coefficient is also highly dependent on the number of layers, with monolayer materials exhibiting the higher expansion coefficient. We have also directly demonstrated that monolayer graphene has a negative thermal expansion coefficient, as previously suggested.[4] Figure 1 shows the thermal expansion coefficient for 2-dim $\mathrm{MoS}_{2}$ and it can be directly seen that the spatial resolution of these measurements is close to the theoretical limit based on the delocalization of the plasmon peak signal. The spatial resolution of the HAADF images, obtained simultaneously with the low-loss EELS maps, remains close to $1 \AA$ at elevated temperatures.

Up to now, window layers in in-situ liquid stages mostly consist of $\mathrm{Si}_{3} \mathrm{~N}_{4}$, which is inert to most reactions within the liquid cell, and, more recently, graphene. While the strong interaction between graphene and the radicals, formed as the results of electron-beam induced radiolysis, lowers the formation of hydrogen bubbles within the liquid, this interaction will also alter the chemical reactions within graphene liquid cells. Therefore, we have developed liquid cells based on 2-dim h-BN layers and will demonstrate that the sensitivity of the liquid to the electron beam is dramatically different (Figure 2). We will also discuss the 
possibility of using such cells for novel EELS experiments including ultra-high resolution low-loss EELS using monochromated electron sources [5].

\section{References:}

[1] B. Standley et al., Nano Lett. 8 (2008), p. 3345.

[2] C. Wang et al., Appl. Mat. 26 (2014), p. 3410.

[3] X. Hu et al, Phys. Rev. Lett. 120(5) (2018), 055902.

[4] C. Wang et al, Adv. Mat, 28(35) (2016), p. 7716.

[5] This work was in part supported by the National Science Foundation (EFMA-1542864 (EFRI))

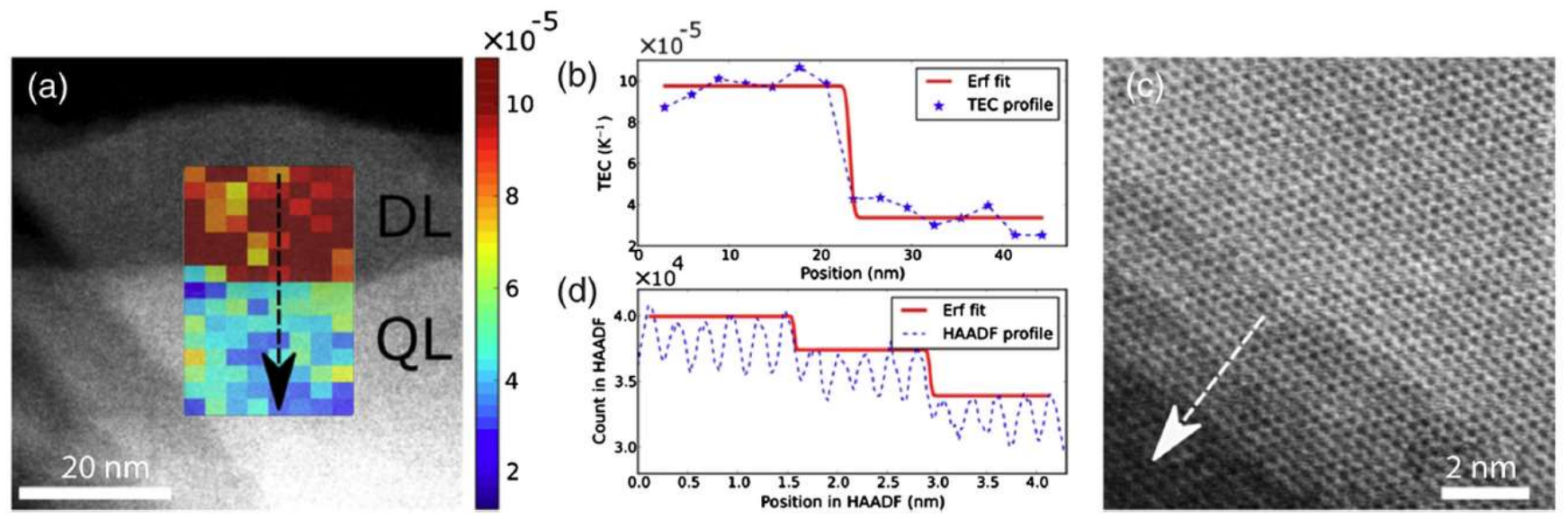

Figure 1. (a) HAADF image of $\mathrm{MoSe}_{2}$ at $623 \mathrm{~K}$ and the spatially resolved map of the local thermal expansion coefficient at the edge a between double-layer (DL) and four-layer (QL) area. (b) Line profile of the thermal expansion coefficient of the interface indicated by the black line in (a). (c) Representative atomic-resolution HAADF image of $\mathrm{MoS}_{2}$ taken at $573 \mathrm{~K}$. (d) Line profile of image contrast across several layers of $\mathrm{MoS}_{2}$ at $573 \mathrm{~K}$.
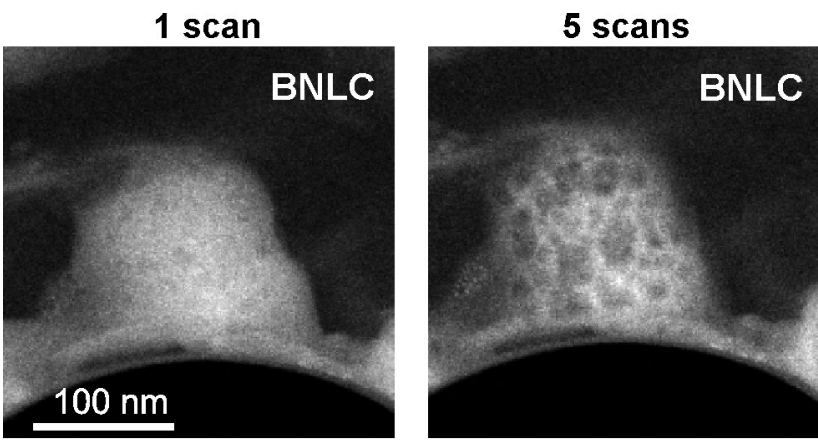

Figure 2. Comparison of the dose rate damage threshold for boron nitride liquid cells (BNLC) and graphene liquid cells (GLC). For the same electron dose-rate (flux) is the same for both liquid cells. The $\sim$ liquid contained in the BNLC show a higher 穴 sensitivity towards the electron beam compared to GLC.
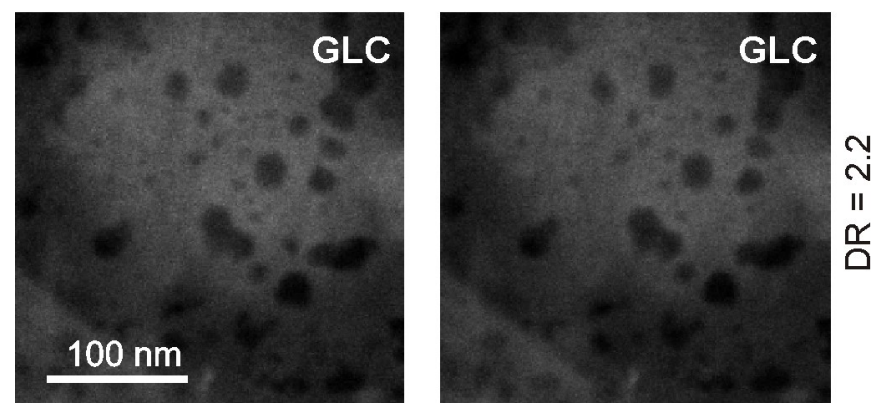